\title{
Técnicas de procesamiento de lenguaje natural en la inteligencia artificial conversacional textual
}

DOI: https://doi.org/10.33262/ap.v3i4.1.123

\begin{abstract}
(c) (i) ()
C.

Natural language processing techniques in textual conversational artificial intelligence
\end{abstract}

Ricardo Javier Celi-Parraga. ${ }^{1}$, Eleanor Alexandra Varela-Tapia. ${ }^{2}$, Iván Leonel AcostaGuzmán. ${ }^{3}$ \& Nestor Rafael Montaño-Pulzara. ${ }^{4}$

\begin{abstract}
Introduction: the internet is advancing at every moment changing the technological landscape of virtual interaction or communication, forcing companies and industries to venture into improving the experience of their customers, which is not only in having websites, social networks and others, but it also leads to improve communication channels and how to interact with each of them. Chatbots allow customers to interact with companies anywhere and at any time, which solves the main problem of costs of call centers or schedules of attention by WhatsApp messages. By adding artificial intelligence based on natural language processing, textual interaction is improved, going from programmed responses to understanding the intention of a user, regardless of the fact that the wording is not specific to our database. Objective: this article aims to analyze bibliographic information on the different AI programming techniques based on NLP and applied to chatbots for textual conversations. Methodology: being literature review research, it is framed in the qualitative methodology, seeking relevant data on the topic

1 Universidad Técnica Luis Vargas Torres de Esmeraldas, Sede Santo Domingo, Ecuador, email: ricardo.celi@utelvt.edu.ec, ID de ORCID https://orcid.org/0000-0002-8525-5744

2 Universidad de Guayaquil, Guayaquil, Ecuador, email: eleanor.varelat@ug.edu.ec, ID de ORCID https://orcid.org/0000-0002-5357-4046

3 Universidad de Guayaquil, Guayaquil, Ecuador, email: ivan.acostag@ug.edu.ec, ID de ORCID https://orcid.org/0000-0002-1589-1825

${ }^{4}$ Sociedad Ecuatoriana de Estadística, Investigador independiente, Ecuador, email: nestor.montano@seeec.org, ID de ORCID https://orcid.org/0000-0001-6813-0029
\end{abstract}


of study. Results: the analysis of the different techniques within the creation of chatbots and their implementations will be presented. Conclusions: a complete analysis of the technique with the best benefits at the moment of creating an intelligent agent capable of maintaining a conversation in natural language and interpreting the users' intention will be presented.

Keywords: ChatBot, NLP, AI Textual conversation, Natural language processing.

\section{Resumen}

Introducción: el internet avanza a cada instante cambiando el panorama tecnológico de la interacción virtual o de comunicación, obligando a las empresas e industrias a incursionar en mejorar la experiencia de sus clientes, lo cual no va solo en tener páginas web, redes sociales y demás, si no que orilla a mejorar los canales de comunicación y como se interactúa por cada uno de ellos. Los chatbots permiten que los clientes se relacionen con las empresas donde sea y a la hora que sea, con lo que se resuelve el principal problema de costos de los call center u horarios de atención por mensajes de WhatsApp. Al agregar inteligencia artificial basado en procesamiento natural del lenguaje se mejora la interacción textual pasando de respuesta programadas a comprender la intención que mantiene un usuario sin importar que la redacción no sea especifica de nuestra base de datos. Objetivo: el presente artículo pretende analizar información bibliográfica de las diferentes técnicas de programación de IA basada en PLN y aplicado a los chatbot para conversaciones textuales. Metodología: al ser una investigación de revisión bibliográfica está enmarcada en la metodología cualitativa, buscando datos relevantes sobre el tema de estudio. Resultados: se presentarán los análisis de las diferentes técnicas dentro de la creación chatbots y sus implementaciones. Conclusiones: se entregará un análisis completo de la técnica con mejores beneficios al momento de crear un agente inteligente capaz de mantener una conversación en lenguaje natural e interpretar la intención de los usuarios.

Palabras claves: ChatBot, PLN, IA, Conversación textual, Procesamiento del lenguaje natural.

\section{Introducción}

Chatbots, los Chatbots son programas informáticos que permite interactuar a un usuario humano con la tecnología, a través de diferentes formas de expresión como textos o voz, pero su principal ventaja es que la disponibilidad es de 24 horas al día sin ningún tipo de interrupción y puede atender a varios usuarios al mismo tiempo sin problema; su utilización va desde hace varios años atrás donde iniciaron bajo el entorno de atención al cliente, hasta la actualidad que pueden ser partícipes en cualquier actividad empresarial.

Por su alcance se le ha dado varios nombres como Bot conversacional, Asistente virtual, Asistente inteligente, Agente virtual, entre otros calificativos; pero así mismo tienen diferentes grados de complejidad o inteligencias para el desarrollo de sus funciones. Para 
lo cual debemos entender el funcionamiento general del mismo, el proceso inicia cuando un usuario humano interactúa con el Chatbot, la interacción puede ser por voz o por texto; en el presente documento nos centraremos en el desarrollo del proceso mediante textos, bajo este lineamiento el agente virtual lo que realiza internamente es separar los caracteres, transformar en minúsculas, corregir faltas ortográficas lo que conocemos como Procesamiento del Lenguaje Natural (PLN), basado en esta información busca en su programación dicha frase para entregar una respuesta.

Como se mencionó en el párrafo anterior los Chatbot tenían respuestas limitadas en base a su programación específica, pero esto cambia en los últimos tiempos al introducir la inteligencia artificial, con la cual no solo se entrega una respuesta si no que entiende la pregunta sin importar la forma de redacción. Esta motivación de la IA en el texto escrito se ha cubierto utilizando diferentes técnicas de PNL, incluido el uso de algoritmos de aprendizaje automático, principalmente por su escalabilidad, capacidad de aprendizaje y rápido desarrollo, métodos que están basados en reglas o enfoques del conocimiento.

Programación de Lenguaje Natural, con el crecimiento de la información subjetiva, los investigadores del procesamiento del lenguaje natural están cada vez más interesados en desarrollar métodos para extraer automáticamente el conocimiento de estas nuevas fuentes, porque esta demanda surge del estudio de sistemas informáticos efectivos para la comunicación entre humanos y computadoras a través del lenguaje natural. Por tanto, la importancia de la emoción en el lenguaje tiene una subdisciplina en PLN cuyo objetivo es identificar y extraer el contenido subjetivo y emocional del texto, llamado análisis de sentimientos (AS).

Las técnicas textuales conversacionales desarrolladas en el marco del curso de Inteligencia Artificial; lo que permite tener una mejor comprensión y una mejor gramática textual. Considerando la complejidad existente del procesamiento del lenguaje natural, se detallan el mecanismo de búsqueda y el sistema de reglas de generación. Se especifica un algoritmo de reprocesamiento especialmente diseñado para simplificar el alcance de la discusión del agente.

En la actualidad, el análisis de texto se realiza de manera automática, en donde la intervención de las computadoras se basa a que el ordenador asimile dichas características de los textos, por ende, la metodología computacional es responsable del análisis de textos, a esto le llamamos Procesamiento del Lenguaje Natural y es utilizado para la obtención de palabras claves dentro de un grupo de documentos, de forma que busca palabras que se repitan dentro de ese apartado, las cuales, no son de suma frecuencia al momento de considerar el total de los documentos (Villena \& Dunstan, 2019).

La documentación con la información procesada y presentada es el principal inconveniente que se presenta al momento de realizar la extracción de la misma, esto se da al no contar con un marco definido o no se encuentra distribuido bajo parámetros previamente establecidos, esto nos lleva a extraer información duplicada, lo que resulta en un incumplimiento de la demanda esperada. Por lo tanto, es recomendable tener un marco definido para la facilitación de obtener los conocimientos de un dominio y 
analizarlos de forma pausada y así poder crear un nuevo proyecto (Marín-Álvarez et al., 2020) .

Según explica Cárdenas y Castillo (2018) los procesamientos de textos estructurados se desarrollan a través de modelos de detección de semejanzas en los códigos de fuentes, el cual se los utilizan para determinar la presencia de prácticas de reutilización, usando técnicas asociadas a la lingüística computacional, tales como, minería de datos sobre textos y el procesamiento del lenguaje natural. Esta identificación de semejanzas de códigos nos sirve para varias intenciones, entre las que se puede señalar, es el estudio de la evolución del código fuente de un proyecto, la detección de plagios o detección de prácticas de reutilización.

Inteligencia artificial conversacional textual, Aristóteles fue considerado uno de los pioneros que definieron una parte las reglas q describen el funcionamiento de la mente para conseguir conclusiones racionales. También nos indica que Ctesibio de Alejandría fue el originario en la construcción de una maquina autocontrolada que permitía regular el flujo de agua, siglos después Alan Turing diseño una máquina universal, el cual exponía la viabilidad de usar un dispositivo físico para el computo formalmente definido. Villalonga (2019) nos brinda su criterio de la inteligencia artificial y los principios de la misma, es una ciencia, en la cual, se aplica el ingenio para crear maquinas inteligentes diseñadas para programas de cómputo, en la época de los griegos ya tenían un pequeño conocimiento de lo que se trabaja la IA.

Estrada (2018) realizo una investigación, la cual fue desarrollada dentro del ambiente de la inteligencia artificial, esto impulsó una búsqueda inteligente para razonar como un ser humano y este simula una conversación con la intención de hacer creer al usuario que está dialogando con otra persona entablando un dialogo amigable, de la idea de este proyecto salió el diseño de un sistema web de búsqueda inteligente conversacional para la ubicación de empresas y servicios, en el cual, el agente inteligente interactúa con los usuarios a través de conversaciones, y los usuarios definían los servicios que desean obtener, esa información es brindaba de acuerdo a los intereses profesionales de cada usuario, este agente genera diálogos creíbles y dinámicos en un lenguaje natural, expresando diálogos coherentes y con la información requerida.

Con la visión por computadora y el procesamiento del lenguaje, la inteligencia artificial débil puede centrarse en la automatización de procesos para aprender fácilmente patrones en los datos que se le proporcionan como por ejemplo para jugar al ajedrez, hacer sugerencias de compra, realizar preferencias de inversión, facilitar la predicción de ventas, el pronóstico del tiempo y, en general, las actividades basadas en patrones que pueden perfeccionarse (Boden, 2017)

Según Munaka (1998) algunas definiciones tradicionales se refieren a la capacidad de un programa de computadora para operar de la misma manera que la mente humana realiza su proceso de aprendizaje y reconocimiento, esta definición se basa en la psicología clásica, la tecnología de búsqueda y el procesamiento del lenguaje natural. 
La evolución tecnológica es nuestra vida, algunos de ellos son agentes de diálogo llamados chatbots o asistentes virtuales, que se desarrollan a través del procesamiento del lenguaje natural, que es una rama de trascendencia de la inteligencia artificial. Los agentes de diálogo o chatbots son programas informáticos diseñados para simular un diálogo inteligente con uno o más usuarios, e incluso con otros sistemas con características similares detrás de los métodos de texto (Orozco et al., 2020). Estos sistemas no solo están diseñados para imitar el diálogo humano o comprender a los usuarios, sino que también tienen muchas aplicaciones prácticas.

Empresas internacionales de renombre como Microsoft, Google, Facebook, IBM y Amazon están invirtiendo en esta tecnología ChatBots y desarrollando e implementando sus propios marcos para ello. En la actualidad, el uso de esta tecnología en los países de América Latina ha crecido enormemente, en países como Chile y Argentina, desde 2019, esta tecnología se ha implementado en los sectores de banca, recursos humanos, comercio electrónico y salud (Orozco et al., 2020).

Los chatbot expresan en el ámbito de la inteligencia artificial de que no solo se encuentran ligados a mensajes de texto sino a que usa técnicas de procesamiento de lenguaje natural, también los chatbots son aplicables en muchos campos como son: educación, viajes, web, servicio al cliente y compras ya que son capaces de tener conversaciones con razonamiento basados en diálogos con el ser humano (Ganan, 2021)

Con estas técnicas permiten a los computadores actuar, por ende, un chatbot es un software que procesa mensajes estructurados para mantener una conversación mediante el lenguaje natural con un humano, a su vez tienen la capacidad de diferenciar entre palabras y emoticones para poder cumplir estas funciones específicamente está programada para que interprete las conversaciones

\section{Metodología}

En la investigación presente se usa una metodología cualitativa la cual, en palabras de Herrera-Enríquez et al. (2021) es usada para el análisis de datos referente al procesamiento del lenguaje natural textual y las técnicas que se pueden usar. Se realiza una indagación en documentos que encontramos en los diferentes repositorios de artículos científicos. En la búsqueda se ha seleccionado en base a los temas Inteligencia Artificial Textual y Técnicas de PLN en la inteligencia artificial conversacional textual

La metodología es utilizada en el estudio de problemas que no se encuentra claramente definido, esta técnica se la utilizó para realización de las investigaciones de los temas de Inteligencia Artificial en reconocimiento del Lenguaje Natural (PLN) textual y sus técnicas, recopilando información en los distintos sitios web (Google académico, Scielo.org, Dialnet.Unirioja.es, Redalyc.org, Sciencedirect.com) (Herrera-Enríquez et al., 2021).

En palabras de Enríquez et al. (2021) esta metodología nos permite visualizar los registros donde obtuvimos la información necesaria, para la compilación de recursos necesarios 
para el desarrollo del articulo propuesto, y de esta manera crear premisas que sirvan como sustento o explicación de manera generalizada.

\section{Resultados}

El procesamiento del lenguaje natural (PLN) es una rama de la inteligencia artificial que ha ganado protagonismo en mundo cada vez más digital, es gracias a las técnicas de PLN que actualmente se pueden realizar traducciones automáticas de texto, revisión de ortografía o conteo de palabras. Según Zeroual (2018) este proceso tiene como objetivo "aprender, comprender, reconocer y producir contenido de lenguaje humano". En sí, trata de mejorar una comunicación eficiente entre el humano y la maquina mediante el uso de lenguas naturales como español, inglés, francés, etc.

Entre las múltiples aplicaciones que ofrece el PLN, una de las más llamativa son los sistemas conversacionales, o también conocidos como "chatbots", los cuales buscan lograr una comunicación más directa y natural con el hombre, pues hasta hace unos años el único medio de interacción con la computadora era a través de dispositivos de entrada y salida como teclado o mouse, por ende, esto no suponía un canal de comunicación interactivo y eficiente (Zeroual, 2018).

El tema de costos es uno de los principales detonantes en la innovación, como en cualquier tema sea de inversión pública o privada la mejora continua debe ser encaminada al uso de tecnologías que ayuden a la reducción de costos (Villalba et al., 2021). Sánchez et al. (2021) por otro lado establece que los costos deben ser encaminado al desarrollo de la población la misma que ayudará con el avance de conocimientos, el desarrollo de inteligencia artificial coadyuva al incremento de conocimiento y economía de la colectividad.

Blanco (2016), en su trabajo "Extracción de Datos Enlazados desde Información no estructurada Aplicando Técnicas PLN y Ontologías" menciona las técnicas PLN, la cual ofrece la posibilidad de utilizarla para una ontología, permitiendo incrementar la extracción de información del texto, consintiendo la extracción de otros conceptos para ampliar la investigación asociada a las entidades. Entre las técnicas utilizadas en este proyecto son:

- Preprocesamiento de datos: En esta fase, el contenido de los ficheros de entradas es clasificado partiendo de la extracción de texto plano utilizando bibliotecas desarrolladas para este propósito, luego, se identifica el texto en qué tipo de idioma se encuentra (inglés o español), a partir de esta tarea se toma la decisión de cuáles serán los patrones a utilizarse para la extracción de conceptos.

- Análisis Sintáctico Superficial: Este se encuentra dirigido inicialmente a la identificación de conceptos, la cual, reside en agrupar los tokens de la oración en chunks que representan las estructuras gramaticales, que se clasifican como: sintagmas nominales, adjetivales, preposicionales, grupos verbales, entre otros, a partir de los cuales se obtiene un árbol sintáctico. 
- Análisis de Dependencia: En esta establecen las relaciones de dependencias concurrentes entre las estructuras gramaticales, las cuales son simbolizadas en un árbol de dependencias. Estos resultados se analizados no solo son tan útiles para emparejar vínculos entre los conceptos presentes en las diferentes estructuras gramaticales, sino también para identificar o construir las frases-enlace a utilizar en el etiquetado de esas relaciones.

Gate es un software que permite solucionar el problema de procesamiento de textos, esta herramienta fue desarrollada por Universidad de Sheffield en 1995, está basada en Java como una arquitectura que define la organización y las responsabilidades. Siendo un Framework que proporciona componentes de procesamiento, que ayudan a disminuir el tiempo de desarrollo de un api y una interfaz, esto incluye recursos para algoritmos y estructura de datos (Alvarez, 2021).

RapidMiner es la herramienta utilizada en la minería de datos que fue desarrollada para Java, permitiendo el impulso de procesos de análisis de datos, usando encadenamiento de 500 operadores mediante un entorno gráfico, el cual permite utilizar los algoritmos incluidos en Weka, este contiene métodos de preprocesamiento de datos, modelación predictiva y descriptiva, métodos de entramientos y prueba de modelos (Jaramillo, 2015)

Weka es otra de las herramientas desarrolladas para Java, permite el aprendizaje automático y minería de datos, es de distribución de licencia GNU-GLP, contiene un amplio catálogo de algoritmos para el análisis de datos y modelado predictivo, permitiendo visualizar los datos a través de su interfaz gráfica. El programa tiene tres entornos gráficos y un entorno en modo consola, lo que permite la implementación de algoritmos diseñados para preprocesamiento, clasificación, agrupación y selección de atributos de datos (Jaramillo, 2015).

El grupo de trabajo Viltres et al. (2018), habla de las técnicas de PLN que fueron utilizadas en su proyecto "Procesamiento Semántico de Información en Sistemas de Recuperación de Información”, las cuales son:

- Recuperación de información: En esta se utiliza la web semántica, es una tecnología generada para los usuarios de internet, la cual nos permite obtener información de buena calidad en la que su información está bien definida, facilitando a los ordenadores trabajar mejor en conjunto a los usuarios, el objetivo principal es permitir que los datos almacenados en esta Web consigan ser procesados por las máquinas de manera inteligente, proporcionando a las personas la búsqueda, integración y análisis de la información que se encuentra disponible.

- Búsqueda semántica de información: El objetivo de la búsqueda semántica es mejorar la exactitud de investigación a través de la comprensión del usuario cuando realiza una consulta y el significado contextual de los datos, la búsqueda semántica predice lo que él usuario expresa abiertamente y se ajusta a la necesidad (contexto), la información es seleccionada con exactitud para el usuario. 
Soroa (2017), en su proyecto menciona a la técnica Recuperación y procesado de documentos que es un método con similitud a las antes ya mencionados, en la cual se manipula la información de los documentos se obtuvieron, todos estos documentos se lo realizo a través de búsquedas en base de datos o en internet, estos son clasificados con direccionamiento de textos o agrupación de textos similares. Estas técnicas trabajan con todo el documento (párrafo o secciones completas), esto documentos tiene un procesado básico de textos.

La recuperación de información y la visualización son aspectos que son mencionados en el área de la inteligencia artificial en cual se encuentra en constante desarrollo debido al continuo aumento de datos generados y almacenados en la web. El proyecto realizado planteo el bosquejo de agentes inteligentes capaces de recuperar y obtener información semiestructurada o no estructurada, esta información obtenida, al ser textual y residir en el lenguaje natural, es procesada con las técnicas de Procesamiento del Lenguaje Natural y el Aprendizaje automático, el cual nos permite hallar los patrones, tendencias y relaciones que existan en el contenido almacenado, para luego ser mostrados al usuario a través visualizaciones interactivas (Medrano, 2020).

La Recuperación de información es el proceso en el que intervienen el análisis de la documentación textuales y las solicitudes de información de los usuarios en el lenguaje natural, estos aspectos se relacionan con efectividad dentro del proceso de recuperación. Las técnicas de recuperación de información facilitan la utilización de bibliotecas de componentes de software, la utilización de estas técnicas de PLN destaca las aproximaciones basadas en la sintaxis y las semánticas (Buenaga, 2004).

Andino y Tapia (2020) hablan de las técnicas de PLN que son utilizadas para la comparación de textos de diferente origen con el propósito de determinar si ambos textos pertenecen a una misma instancia de una entidad, una de estas técnicas es basadas en el análisis del deletreo y distancias, esta técnica se encarga de identificar la diferencia que existe entre las cadenas de caracteres, estas diferencias se generan por errores al momento de la digitalización, errores tipográficos de inserción, de supresión, o modificación de caracteres. La técnica no necesita diccionarios de variantes, ya que se establece mediciones basadas en la diferencia de caracteres en la cadena

Delgado (2015) menciona en su trabajo "Hacia un algoritmo Optimo de Emparejamiento de nombres", tres tipos de técnicas (fonéticas, de deletreo y distancia, y combinadas) en la cual nos enfocamos en el deletreo y distancia. La cual genera un valor máximo de similitud parecido al valor mínimo de distancia, esto dando como resultado de las operaciones de inserción, borrado o inserción de caracteres para comparar dos palabras. Otras técnicas de deletreo se relacionan con el reconocimiento de patrones de texto y la segmentación de palabras en sub - unidades en $\mathrm{N}$ caracteres ( $\mathrm{N}$-gramas).

En base a los análisis de los diferentes autores presentamos en la tabla 1 un resumen de las principales técnicas al momento de programar 


\section{Tabla 1}

Técnicas de PLN en la IA

\begin{tabular}{|c|c|c|}
\hline Técnicas & Función & Objetivo \\
\hline Preprocesamiento de datos & $\begin{array}{l}\text { Se basa en el contenido de los } \\
\text { ficheros de entradas clasificado } \\
\text { utilizando } \\
\text { desarrolladas } \\
\text { propósito. }\end{array}$ & $\begin{array}{l}\text { Toma la decisión de cuáles serán } \\
\text { los patrones a utilizarse para la } \\
\text { extracción de concepto }\end{array}$ \\
\hline Análisis Superficial & $\begin{array}{l}\text { Está dirigido inicialmente a la } \\
\text { identificación de concepto }\end{array}$ & $\begin{array}{l}\text { Representan las estructuras } \\
\text { gramaticales }\end{array}$ \\
\hline Análisis de Dependencia & $\begin{array}{l}\text { Establecen las relaciones de } \\
\text { dependencias concurrentes entre } \\
\text { las estructuras gramaticales. }\end{array}$ & \begin{tabular}{lll} 
Diferencia & & \multicolumn{2}{c}{ estructuras } \\
gramaticales e identifica o & \\
construye & &
\end{tabular} \\
\hline Recuperación de información & $\begin{array}{l}\text { Análisis de información textual } \\
\text { ingresada por los usuarios } \\
\text { basados en sintaxis y semánticas }\end{array}$ & $\begin{array}{l}\text { Obtención de información de } \\
\text { buena calidad para tratar mejor } \\
\text { en conjunto a los usuarios }\end{array}$ \\
\hline $\begin{array}{l}\text { Búsqueda semántica } \\
\text { información }\end{array}$ & $\begin{array}{l}\text { La búsqueda semántica predice } \\
\text { lo que los usuarios expresan } \\
\text { públicamente y lo ajusta según } \\
\text { sea necesario }\end{array}$ & $\begin{array}{l}\text { Mejora de investigación y se } \\
\text { ajusta a la necesidad del usuario }\end{array}$ \\
\hline $\begin{array}{l}\text { Técnicas basadas en el análisis } \\
\text { del deletreo y distancia }\end{array}$ & $\begin{array}{l}\text { Se encarga de identificar la } \\
\text { diferencia que existe entre las } \\
\text { cadenas de caracteres. }\end{array}$ & $\begin{array}{l}\text { Mediciones basadas en la } \\
\text { diferencia de caracteres en la } \\
\text { cadena }\end{array}$ \\
\hline
\end{tabular}

El análisis del agente de diálogo se puede realizar simplemente mediante un proceso de comparación con una lista de frases explicativas. Por tanto, esta no es una solución completa por lo que se debe interactuar con el uso de herramientas informáticas que ayuden a mejorar la búsqueda de información, se plantea un gran desafío para la interpretación y el procesamiento del lenguaje natural y la optimización del tiempo de respuesta de los agentes.

La recuperación de información es la base del proceso que se realiza en lenguaje natural, para lo cual se pueden utilizar diferentes modelos para identificar y marcar entidades que serán comparadas y clasificadas en dos o más categorías. Debido al creciente número de documentos electrónicos en las bibliotecas digitales, la clasificación y clasificación de textos son los temas más estudiados en el procesamiento del lenguaje natural. Como métrica para comparar el rendimiento global de diferentes algoritmos en el conjunto de datos de entrenamiento etiquetado, se han recopilado informes de diferentes autores, lo que demuestra que es fundamental involucrar la inteligencia artificial en estos procesos.

\section{Conclusiones}

- Como resultado principal de la presente investigación, se detallaron las principales técnicas de programación para de chatbot describiendo las herramientas de procesamiento del lenguaje natural más potentes.

- El uso de tecnologías cognitivas enfocadas a IA es un punto clave que facilita el desarrollo de agentes virtuales o chatbots porque se encargan de procesar automáticamente el lenguaje natural, identificar las necesidades del usuario y 
permitir enfocarse en la implementación de reglas de negocio.

- Con la misión de no interrumpir la dinámica de las interacciones usuario-agente, las estrategias de inteligencia artificial ayudan a superar los inconvenientes asociados con la reducción del tiempo. El análisis del lenguaje natural y la definición de gramática; simplifican la base de conocimiento del agente. Produce un vocabulario que consta de un conjunto reducido de palabras clave, lo que reduce el espacio de búsqueda y hace que el proceso sea más rápido y eficaz.

- Estas tecnologías han promovido el desarrollo de lenguajes naturales gramaticales, permitiéndoles enfocarse en todos los aspectos de estos comportamientos de agencia. De esta forma, con la ayuda de estas tecnologías de inteligencia artificial, se ha avanzado en el desarrollo de agentes inteligentes que puedan llevar a cabo conversaciones sobre estos temas en lenguaje natural. A partir del uso de un algoritmo de preprocesamiento especialmente diseñado, puede determinar si puede dar una respuesta a la frase ingresada por el usuario. Mediante el uso de la interfaz, la función del chatbot se oculta, lo que evita que los usuarios distingan la naturaleza de otros participantes en la conversación.

\section{Referencias bibliográficas}

Alvarez, N. (2021). Mapeo Sistemático Sobre Los Métodos, Técnicas Y Tecnologías Orientadas A La Detención De Noticias Falsas. Ecuador - PUCESE - Escuela de Sistemas y Computación.

Andino, J., \& Tapia, L. (2020). Social media como medio de análisis y prevención del acoso cibernético, aplicado a los perfiles de Facebook, utilizando técnicas de procesamiento de lenguaje natural con base en Levenshtein. Universidad de las Fuerzas Armadas ESPE. Obtenido de http://repositorio.espe.edu.ec/bitstream/21000/23755/1/T-ESPE-044273.pdf

Blanco, A. R. (2016). Extracción de Datos Enlazados desde Información No Estructurada Aplicando Técnicas de PLN y Ontologías. In XIV Congreso Internacional de Información Info'2016.

Boden, M. (2017). Inteligencia artificial. Turner.

Buenaga, M. (2004). Integración de técnicas de procesamiento del lenguaje natural para la recuperación de información en bibliotecas de componentes software. Universidad Complutense de Madrid, Facultad de Ciencias Físicas. Obtenido de https://eprints.ucm.es/id/eprint/1960/

Cárdenas, M., \& Castillo, J. (2018). Procesamiento de Textos estructurados. In $x x$ Workshop Investifadores en Ciencias de la Computación. Universidad Nacional de Nordeste. Obtenido de http://sedici.unlp.edu.ar/handle/10915/67125

Delgado, J. (2015). Hacia un Algoritmo Optimo de Emparejamiento de Nombres. Revista de Analisis Estadistico/Journal of Statistical Analysis, 9(1), 97. 
Enríquez, G. H., Páez, S. C., Vera, D. Z., Sánchez, M. J. H., \& Villalba, C. I. C. (2021). Incidencia de las metodologías de enseñanza en las carreras de ciencias administrativas ofertadas por las universidades públicas del DMQ. Visionario Digital, 5(1), 6-25.

Estrada, L. (2018). Implementar chatbot basado en inteligencia artificial para la gestión de requerimientos e incidentes en una empresa de seguros. Universidad San Ignacio de Loyola. Obtenido de http://repositorio.usil.edu.pe/handle/USIL/8844

Ganan, R. (2021). Diseño y construcción de un chatbot basado en machine learning: caso de estudio asistente virtual para maestrías Utmach. Universidad Técnica de Machala.

Herrera-Enríquez, G., Herrera-Sánchez, M., Casanova-Villalba, C., Puyol-Cortez, J., \& Mendoza-Armijos, H. (2021). Manual para Elaboración del Plan de Titulación como Conclusión de Carrera. Grupo Compas.

Jaramillo, A. (2015). Aplicación de técnicas de minería de datos para determinar las interacciones de los estudiantes en un entorno virtual de aprendizaje (Vol. 28). Revista Tecnológica - ESPOL.

Marín-Alvarez, D., Manrique-Losada, B., \& Quintero, J. (2020). Método para la representación semi-automática de modelos conceptuales desde documentos de negocio escritos en lenguaje natural en español. Ingeniare. Revista Chilena de Ingeniería, 28(4), 565-584. doi:http://dx.doi.org/10.4067/S071833052020000400565

Medrano, J. (2020). Agentes inteligentes para recuperación de información y analítica visual en big data. In XXII Workshop de Investigadores en Ciencias de la Computación. Obtenido de http://sedici.unlp.edu.ar/handle/10915/103444

Munaka, T. (1998). Fundamentals of the new Artificial Intelligence: beyond Traditional Paradigms. Springer.

Orozco, M., Panizza, L., Vegega, C., Pytel, P., \& Pollo, M. (2020). Metodología de implementación de un ChatBot como tutor virtual en el ámbito educativo. In XXII Workshop de Investigadores en Ciencias de la Computación .

Sánchez, M. J. H., Villalba, C. I. C., Armijos, H. E. M., Requelme, S. E. R., \& Farías, J. J. C. (2021). El Crédito de Desarrollo Humano como estrategia de la Economía Popular y Solidaria para combatir la pobreza. Visionario Digital, 5(1), 52-69.

Soroa, A. R. (2017). Plataformas y sistemas de procesamiento lingüístico de alto rendimiento. Estudio preparado por la UPV-EHU para el Plan TL.

Villalba, C. I. C., Sánchez, M. J. H., Zambrano, C. M. N., \& López, S. E. R. (2021). Modelo de calidad para el mejoramiento de la eficiencia en las instituciones públicas del Ecuador. Ciencia Digital, 5(1), 15-29. 
Villalonga, E. (2019). Inteligencia artificial y el internet de las cosas: estudio y estado actual en empresas y consumidores. Universidad de Valladolid. Obtenido de Inteligencia artificial y el internet de las cosas: estudio y estado actual en empresas y consumidores: https://core.ac.uk/download/pdf/232122917.pdf

Villena, F., \& Dunstan, J. (2019). Obtención automática de palabras claves en textos clínicos: una apliación de procesamiento del lenguaje natural a datos masivos de sospecha diagnóstica en Clile. Revista médica de Chile, 147(10), 1229-1238.

Viltres, S., Rodríguez, P., Febles, J., \& Estrada, S. (2018). Semantic processing of information in Information Retrieval Systems. Revista Cubana de Ciencias Informáticas, 12(1), 102-116.

Zeroual, I. \&. (2018). Data science in light of natutal language processing: An overview. Procedia computer science, 127 , 82-91. doi:https://doi.org/10.1016/J.PROCS.2018.01.101 


\section{PARA CITAR EL ARTÍCULO INDEXADO}

Celi-Parraga, R. J. . ., Varela-Tapia, E. A., Acosta-Guzmán, I. L., \& Montaño-Pulzara, N. R. (2021). Técnicas de procesamiento de lenguaje natural en la inteligencia artificial conversacional textual . AlfaPublicaciones, 3(4.1), 40-52. https://doi.org/10.33262/ap.v3i4.1.123

\section{Ciencia}

El artículo que se publica es de exclusiva responsabilidad de los autores y no necesariamente reflejan el pensamiento de la Revista Alfa Publicaciones.

El artículo queda en propiedad de la revista y, por tanto, su publicación parcial y/o total en otro medio tiene que ser autorizado por el director de la Revista Alfa Publicaciones.
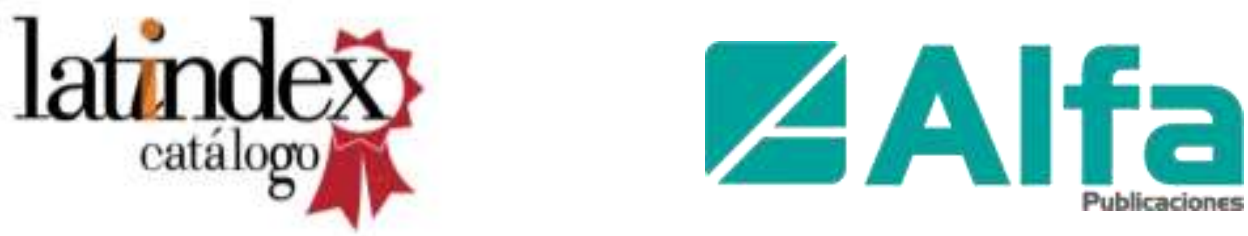